\title{
DUSP6 regulates drug sensitivity by modulating DNA damage response
}

\author{
TV Bagnyukova ${ }^{1}$, D Restifo ${ }^{1}$, N Beeharry ${ }^{1}$, L Gabitova ${ }^{1}$, T Li ${ }^{2}$, I G Serebriiskii ${ }^{1}$, E A Golemis ${ }^{1}$ and I Astsaturov ${ }^{\star}, 1$ \\ ${ }^{1}$ Program in Developmental Therapeutics, Fox Chase Cancer Center, 333 Cottman Avenue, Philadelphia, PA 19111, USA and \\ ${ }^{2}$ Biostatistics and Bioinformatics Facility, Fox Chase Cancer Center, 333 Cottman Avenue, Philadelphia, PA 19111, USA
}

Background: Dual specificity phosphatase 6 (DUSP6) is a member of a family of mitogen-activated protein kinase phosphatases that dephosphorylates and inhibits activated ERK1/2. Dual specificity phosphatase 6 is dynamically regulated in developmental and pathological conditions such as cancer.

Methods: Cancer cell lines were made deficient in DUSP6 by siRNA and shRNA silencing. Sensitivity to anti-EGFR and chemotherapeutic agents was determined in viability and apoptosis assays, and in xenografts established in SCID mice. Cellular effects of DUSP6 inactivation were analysed by proteomic methods, followed by analysis of markers of DNA damage response (DDR) and cell cycle.

Results: We determined that depletion of DUSP6 reduced the viability of cancer cell lines and increased the cytotoxicity of EGFR and other targeted inhibitors, and cytotoxic agents, in vitro and in vivo. Subsequent phosphoproteomic analysis indicated DUSP6 depletion significantly activated CHEK2 and p38, which function in the DDR pathway, and elevated levels of phosphorylated H2AX, ATM, and CHEK2, for the first time identifying a role for DUSP6 in regulating DDR.

Conclusion: Our results provide a novel insight into the DUSP6 function in regulating genomic integrity and sensitivity to chemotherapy in cancer.

Dual specificity phosphatase 6 (DUSP6, also known as MKP3 and PYST1) belongs to the family of mitogen-activated protein kinase (MAPK) phosphatases (Keyse, 2008; Furukawa, 2009). Dual specificity phosphatase 6 negatively regulates mitogenic signalling emanating from receptor tyrosine kinases (RTKs) such as EGFR (Zhang et al, 2010) and FGFR (Li et al, 2007) and their downstream effector RAS (Bluthgen et al, 2009), in part by inducing the dephosphorylation and inactivation of ERK1/2 (Camps et al, 1998). Consistent with this function, DUSP6 depletion in chick embryos (Eblaghie et al, 2003) and gene knockout in mice (Li et al, 2007) produced phenotypes indicative of increased FGF signalling. Thus, one physiological action of DUSP6 is to limit RTK signalling during development and in cellular homoeostasis.

The DUSP6 function in cancer has proven more elusive, and may vary dependent on the tumour type. Loss of DUSP6 expression due to promoter hypermethylation is associated with the progression from the pancreatic intraepithelial neoplasia to invasive ductal carcinoma, leading to the suggestion that DUSP6 functions in this cancer type as a tumour suppressor (Furukawa et al, 2003). In contrast, elevated DUSP6 expression is associated with poor prognosis in non-small cell lung cancer (Chen et al, 2007). However, DUSP6 is elevated in KRAS mutant colon cancer without an effect on outcomes (De Roock et al, 2009), suggesting a more complex function.

We previously reported the use of a network-based approach in combination with siRNA library screening to identify genes that modulate the sensitivity of cells to EGFR inhibitors that are widely used in the clinic (Astsaturov et al, 2010). As a result of this screen, we discovered that knockdown of DUSP6 potently sensitises A431 and other cancer cell lines to EGFR inhibitors. In subsequent mechanistic analysis reported here, we have now established that DUSP6 depletion has a broader activity in sensitising cells not only to EGFR inhibitors but also to additional targeted agents and

*Correspondence: Dr I Astsaturov; E-mail: igor.astsaturov@fccc.edu

Received 6 February 2013; revised 13 June 2013; accepted 14 June 2013; published online 9 July 2013

(c) 2013 Cancer Research UK. All rights reserved 0007-0920/13 
to DNA-damaging agents. Using a phosphoproteomic approach to probe DUSP6 action, we unexpectedly find that DUSP6 depletion activates the ATM-CHEK2 pathway proteins and increases expression of markers of DNA breaks. These results suggest that DUSP6 may be a valuable biomarker and regulator of responsiveness to a range of targeted and DNA cytotoxic agents.

\section{MATERIALS AND METHODS}

Cell lines, compounds, and antibodies. The A431 cervical adenocarcinoma and HEK-293T cell lines were obtained from the American Type Culture Collection and maintained at the Fox Chase Cancer Center Cell Culture Facility. The identity of A431 cell line was confirmed by genotyping. The FaDu, SCC25, and Detroit cells were obtained from Dr TY Seiwert (University of Chicago, Chicago, IL, USA). All cell lines were maintained in Dulbecco's modified Eagle's medium (DMEM) supplemented with $10 \%$ foetal bovine serum (FBS) and L-glutamine. Cetuximab and erlotinib were purchased from the Fox Chase Cancer Center pharmacy; CPT11 was from Sigma-Aldrich (St. Louis, MO, USA); dasatinib was purchased from LC Labs (Woburn, MA, USA). Antibodies to DUSP6 and pCHEK2 were purchased from R\&D Systems, Inc. (Minneapolis, MN, USA) against pH2AX, ATM, and pATM from EMD Millipore Corporation (Billerica, MA, USA). All other antibodies were obtained from Cell Signaling Technology (Danvers, MA, USA).

Drug sensitivity assay. To test in vitro drug sensitivity, tumour cells were plated in 96-well plates in 1\% FBS/DMEM/L-glutamine with 3000 cells per well and incubated overnight. Drugs were added as $10 \times$ stocks in media, and cell viability was measured in $72 \mathrm{~h}$ with CellTiter Blue Viability Assay (Promega, Madison, WI, USA).

Annexin V and TUNEL assays. Apoptosis was measured with the Annexin $\mathrm{V}$ surface labelling, DNA fragmentation was assessed by the TUNEL assay (both using assay kits from Guava Technologies, Hayward, CA, USA). Cells were plated in 12-well plates, and the next day they were treated with $1 \mu \mathrm{M}$ erlotinib, or $50 \mathrm{~nm} \mathrm{CPT} 11$, or $0.01 \mathrm{mg} \mathrm{ml}^{-1}$ of cetuximab. After $72 \mathrm{~h}$ of incubation, positive cells were counted using Guava flow cytometer.

Phosphoproteomic analysis. Relative levels of protein phosphorylation in A431 cells stably expressing control, DUSP6, or DUSP7 shRNA were assessed using the Human Phosphokinase Proteome Profiler Array (ARY003, R\&D Systems, Inc.), according to the manufacturer's protocol. The kit contains nitrocellulose membranes spotted in duplicates with antibodies against 46 phosphosites of signalling molecules. Cells were grown in DMEM supplemented with $1 \%$ FBS for $48 \mathrm{~h}$, and then treated with $1 \mu \mathrm{M}$ erlotinib or vehicle alone for $3 \mathrm{~h}$, and cell lysates were collected and applied to the blocked membranes. The film images were scanned and analysed with the ImageJ software (NIH, Bethesda, MD, USA). The spot densities of proteins of shDUSP6 or shDUSP7 were normalised to control cell line. For some proteins, their phosphorylation status was validated by western blot.

Western blot. Cell lysates were prepared using M-PER mammalian protein extraction reagent (Thermo Fisher Scientific Inc., Rockford, IL, USA). Protein concentration was determined using a BSA kit (Pierce, Rockford, IL, USA). Proteins were resolved by SDS-PAGE, transferred to PVDF, probed first with primary antibodies, and then with IRDye secondary antibodies (LI-COR Biosciences, Lincoln, NE, USA) suitable for the Odyssey imaging system. Membranes were then scanned using an Odyssey scanner (LI-COR Biosciences) and quantified.
Dual specificity phosphatase 6 silencing. Validated DUSP6_5 siRNA (Astsaturov et al, 2010; Hs_DUSP6_5, SI02627338; Qiagen Inc., Valencia, CA, USA) was used to target DUSP6, and GL2 (targeting an insect luciferase, Thermo Fisher Scientific Inc. (Waltham, MA, USA)) and PLK1 were correspondingly the negative and positive controls for transfection, respectively. Depletion of DUSP6 protein isoforms $a$ and $b$ has been validated by western blot (Figure 1A) and quantitative PCR (Figure 1D). Cells were combined with HiPerFect transfection reagent (Qiagen Inc.) and siRNAs in $1 \%$ FBS-DMEM and after $24 \mathrm{~h}$ followed by treatment with drugs. Sensitivity to drugs and effects of DUSP6 knockdown on DNA damage were assessed by the CellTiter Blue Viability Assay, immunofluorescence, and western blot. A431 were puro-

mycin selected following transduction with a replication-deficient lentiviral vector to express two independent shRNA sequences targeting DUSP6 (shDUSP6_1 and shDUPS6_2), shDUSP7, or non-silencing control shRNA (Thermo Fisher Scientific Inc., Lafayette, CO, USA). Two unique shRNAs targeting DUSP6 mRNA were used: DUSP6_1 5'-(AAACTGTGGTGTCTTGGTAC AT) $-3^{\prime}$ and DUSP6_2 5'-(CCGGCATCAAGTACATCTTGAA)- $3^{\prime}$.

Plasmid transfection. HEK-293T cells were seeded in six-well plates to $50 \%$ confluency and in $24 \mathrm{~h}$ transfected with plasmids mixed with lipofectamine (Life Technologies, Grand Island, NY, USA). In all experiments, equal amounts of empty vector DNA were added to controls. Plasmid pBabe-PuroMEK-DD (Boehm et al, 2007) was obtained from Addgene (Addgene plasmid 15268, Cambridge, MA, USA). Dual specificity phosphatase 6 full-length cDNA was cloned into pLEX-HA-MYC lentiviral vector (Thermo Fisher Scientific Inc., Lafayette, CO, USA). Catalytically inactive DUSP6-C293S mutant was made using site-directed mutagenesis (Stratagene, La Jolla, CA, USA).

Immunofluorescence microscopy. A431 cells were plated on glass slides in DMEM supplemented with $1 \%$ FBS. After $24-48 \mathrm{~h}$ of growth, cells were treated with $100 \mathrm{nM}$ CPT11 for $3 \mathrm{~h}$ or $5 \mu \mathrm{M}$ erlotinib overnight. After that, cells were fixed, washed, permeabilised using 1\% Triton-X100, blocked in 3\% BSA and probed first with a primary antibody, and then with a secondary antibody conjugated with AlexaFluor-568 (Invitrogen, Grand Island, NY, USA). Images were acquired on a Nikon C1 Spectral confocal microscope (Nikon, Melville, NY, USA). Quantification of $\mathrm{pH} 2 \mathrm{AX}$, pATM, and p53BP1 was done by counting cells with $>10$ nuclear foci as positive in randomly chosen fields.

Cell cycle. To study cell cycle progression, cells were synchronised with either thymidine or nocodazole. Double thymidine block synchronises cells at the G1/S transition. Cells plated in six-well plates were incubated with $2 \mathrm{~mm}$ thymidine for $18 \mathrm{~h}$. Then the thymidine was washed out and cells were refed with fresh medium for $10 \mathrm{~h}$ before adding thymidine. After the second 16 -h incubation with thymidine, cells were collected at the indicated time points after release from the thymidine block. To synchronise cells in the G2/M transition, cells were treated with $100 \mathrm{~nm}$ nocodazole for $16 \mathrm{~h}$, released from the block, and collected at the indicated time points. Cells were fixed and stained with propidium iodide, and the DNA content was analysed using the FACS flow cytometry. For investigating the effects of DNA-damaging drugs on the cell cycle progression, we incubated cells with $100 \mathrm{nM}$ CPT11, a compound known to block cells at the $\mathrm{S}$ phase. After $20 \mathrm{~h}$ incubation, the drug was washed out and progression through the cell cycle was monitored for $24 \mathrm{~h}$.

Mouse xenografts. Male CB.17/SCID mice aged 6-8 weeks were obtained from the Fox Chase Cancer Center breeding colony. All experiments were performed according to protocols approved by the institutional animal committee. Mice were 
injected subcutaneously to the flanks with three million of A431 cells stably expressing shRNA-DUSP6 or non-silencing control shRNA. When tumours became palpable and reached $80-100 \mathrm{~mm}^{3}$, mice were allocated randomly into two groups: 10 mice per group; one receiving cetuximab $0.75 \mathrm{mg} \mathrm{kg}^{-1}$ in $0.8 \%$ saline, whereas another treated with saline alone. The mice were treated intraperitoneally twice a week for 3 weeks. Then, the treatment was stopped and the tumour growth was monitored until the tumours exceeded a volume of $2000 \mathrm{~mm}^{3}$ or became ulcerated, or animals demonstrated distress or weight loss $>10 \%$ as per the local IACUC guidelines; after that the mice were euthanised. Tumour volume was calculated using the formula: tumour volume $\left(\mathrm{mm}^{3}\right)=\left(\right.$ smallest diameter ${ }^{2} \times$ largest diameter)/2. Drug efficacy was expressed as the percentage tumour growth inhibition (\%TGI), calculated using the equation $100-(T / C \times 100)$, where $T$ is the mean tumour volume of the treated tumours and $C$ is the mean volume in the control group at the time of killing of the first mouse in the control group. Statistical analysis was performed for the log-transformed tumour volumes.

Quantitative RT-PCR. For evaluation of the target genes' knockdown, cells were transfected in six-well plates and total RNA was extracted using RNeasy Minikit (Qiagen Inc.), 48-72 h after transfection. Detection of isoform $a$ and $b$ was performed with standardised assay primers and probes (Hs00169257_m1 for isoform $a$ and Hs00737962_m1 for isoform b; Ambion, Inc., Austin, TX, USA). Quantitative RT-PCR reactions were performed using TaqMan probes and primers designed by the manufacturer, using an ABI PRISM 7700 detection system (Applied Biosystems, Foster City, CA, USA). The results were analysed using the comparative Ct method to establish relative expression curves.

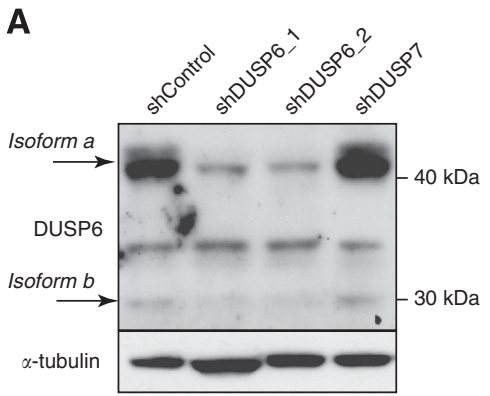

B

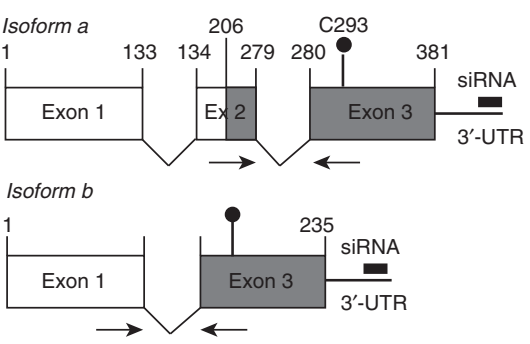

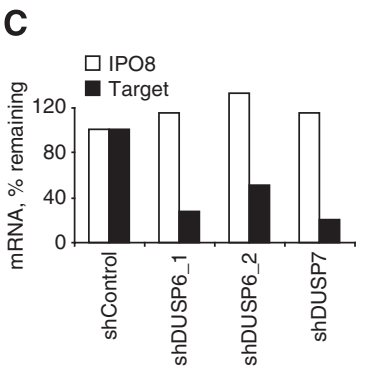

D

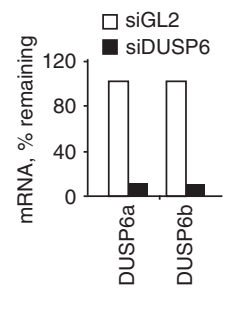

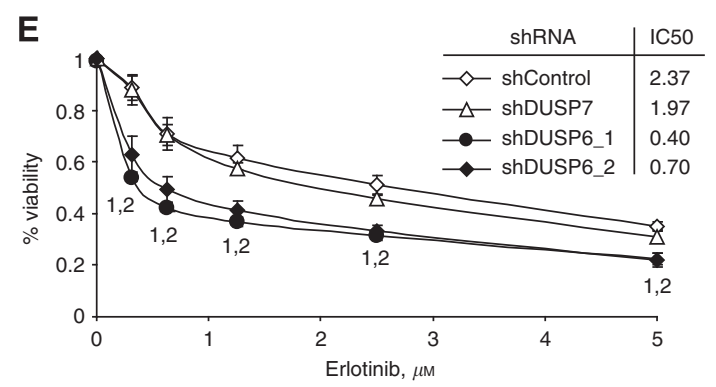

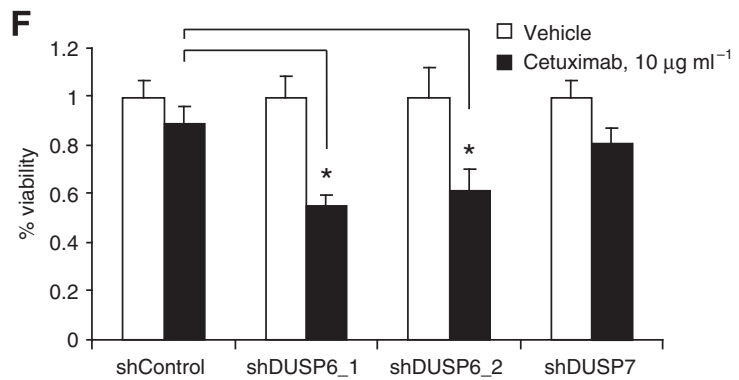

G

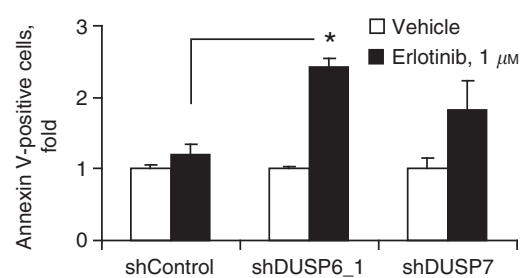

H

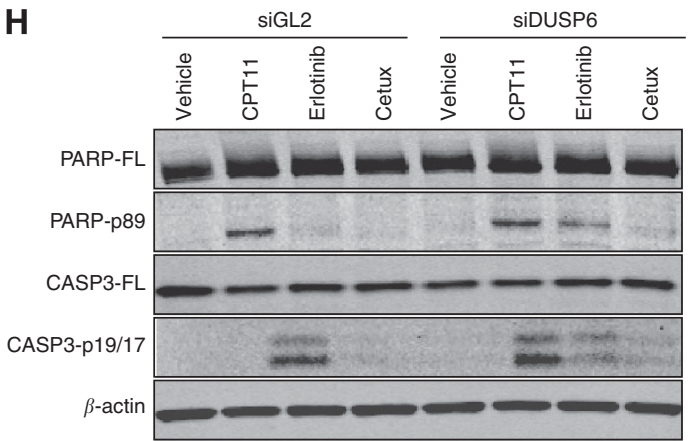

Figure 1. DUSP6 silencing in vitro and in vivo sensitises to EGFR inhibitors. (A) Loss of DUSP6 protein expression in shRNA-modified A431 cells as determined by western blot. (B) Schema of DUSP6 mRNA and protein. Numbers are amino-acid positions, PCR primers are indicated by arrows; thick line shows siRNA DUSP6_5 targeting 3'-UTR, the phosphatase domain is shaded; C293 indicates catalytic cysteine-293. (C) Silencing of DUSP6 and DUSP7 mRNA was confirmed by qPCR in A431 cells stably expressing the indicated shRNA. (D) Silencing DUSP6 with validated siRNA depleted both isoforms of DUSP6 using isoform-specific PCR primers. Shown is percent of remaining mRNA relative to non-targeting GL2 control. $(E, F)$ Silencing of DUSP6 increased the cytotoxicity of EGFR inhibitors, erlotinib (E) and cetuximab (F) in A431 cells. Results are shown as means of four independent experiments \pm s.e.m., shDUSP6 vs non-silencing shRNA control at the corresponding drug concentrations; ${ }^{\star} P$-values (1) shDUSP6_1; and (2) shDUSP6_2 vs non-silencing shRNA control are <0.01, the Wilcoxon's test. Results with shDUSP7 are not statistically significant $(P=0.0976)$. (G) DUSP6 depletion with shRNA enhances apoptosis of A431 cells treated with $1 \mu \mathrm{m}$ erlotinib for $72 \mathrm{~h}$, as measured by the Annexin $V$ staining. Shown are means of four independent experiments \pm s.e.m.; ${ }^{\star} P<0.05$. (H) DUSP6 depletion enhances erlotinib-induced apoptosis in FaDu cells. Cells were made deficient in DUSP6 by siRNA transfection and, 24 later, were treated with $2 \mu \mathrm{m}$ erlotinib, $10 \mu \mathrm{g} / \mathrm{ml}$ cetuximab, 25 nM CPT11 or vehicle for 48 hours. Markers of apoptosis, cleavage of poly-ADP-ribose polymerase (PARP) and caspase 3 (CASP3), were determined by Western blot (shown) in two independent experiments. 


\section{RESULTS}

Dual specificity phosphatase 6 depletion sensitises A431 cells to EGFR inhibitors in vitro and in vivo. Catalytically active DUSP6 (isoform $a$ ) is the predominant isoform of DUSP6 protein expressed in A431 cells (Figure 1A and B). To investigate the mechanism by which DUSP6 affected drug response, we employed lentiviral shRNA vectors for stable depletion of the DUSP6 mRNA in A431 cells. We confirmed depletion by both western blot analysis and quantitative RT-PCR (Figure $1 \mathrm{~A}-\mathrm{C}$ and Supplementary Figure S1A and B). Parallel depletion of the structurally similar DUSP7 phosphatase (Dowd et al, 1998) and use of a non-silencing shRNA served as controls (Figure 1A-C). Consistent with our previously reported siRNA depletion studies (Astsaturov et al, 2010), shRNA depletion of DUSP6 with two independent RNA-targeting sequences significantly increased sensitivity of the A431 cells to the EGFR inhibitor erlotinib, whereas DUSP7 depletion did not (Figure 1E, Supplementary Figure S1C). Similar results were observed with sensitisation to the EGFR inhibitor cetuximab (Figure 1F, Supplementary Figure S1D).
We determined that increased sensitivity to erlotinib of DUSP6depleted cells was associated with increased apoptosis, reflected by increased Annexin V (Figure 1G) and elevated poly-ADP-ribose polymerase and caspase 3 cleavage, and staining (Figure $1 \mathrm{H}$ ).

Importantly, DUSP6 depletion profoundly increased the sensitivity of A431 cells to the inhibition of the EGFR pathway in vivo, based on the analysis of subcutaneously implanted A431 xenografts containing integrated shDUSP6 or control shRNA (Figure 2A, Supplementary Figure S2A). Both control and DUSP6deficient cells formed palpable tumours at 8-10 days after implantation, although shDUSP6 A431 tumours grew more slowly compared with controls (Figure 2A, Supplementary Figure S2A). Treatment of control-depleted tumours slightly delayed growth, and growth resumed after cessation of cetuximab treatment. By contrast, cetuximab treatment suppressed growth of shDUSP6 tumours by $83 \% v s$ controls (TGI $83 \%, P<0.0001$ ), with this effect lasting at least 3 weeks after the last dose of cetuximab (Figure 2A).

Other studies (Zhang et al, 2010) have noted functional interactions between DUSP6 and EGFR, with EGF treatment inducing DUSP6 mRNA expression, erlotinib treatment depressing DUSP6 mRNA expression in some cell types, and DUSP6
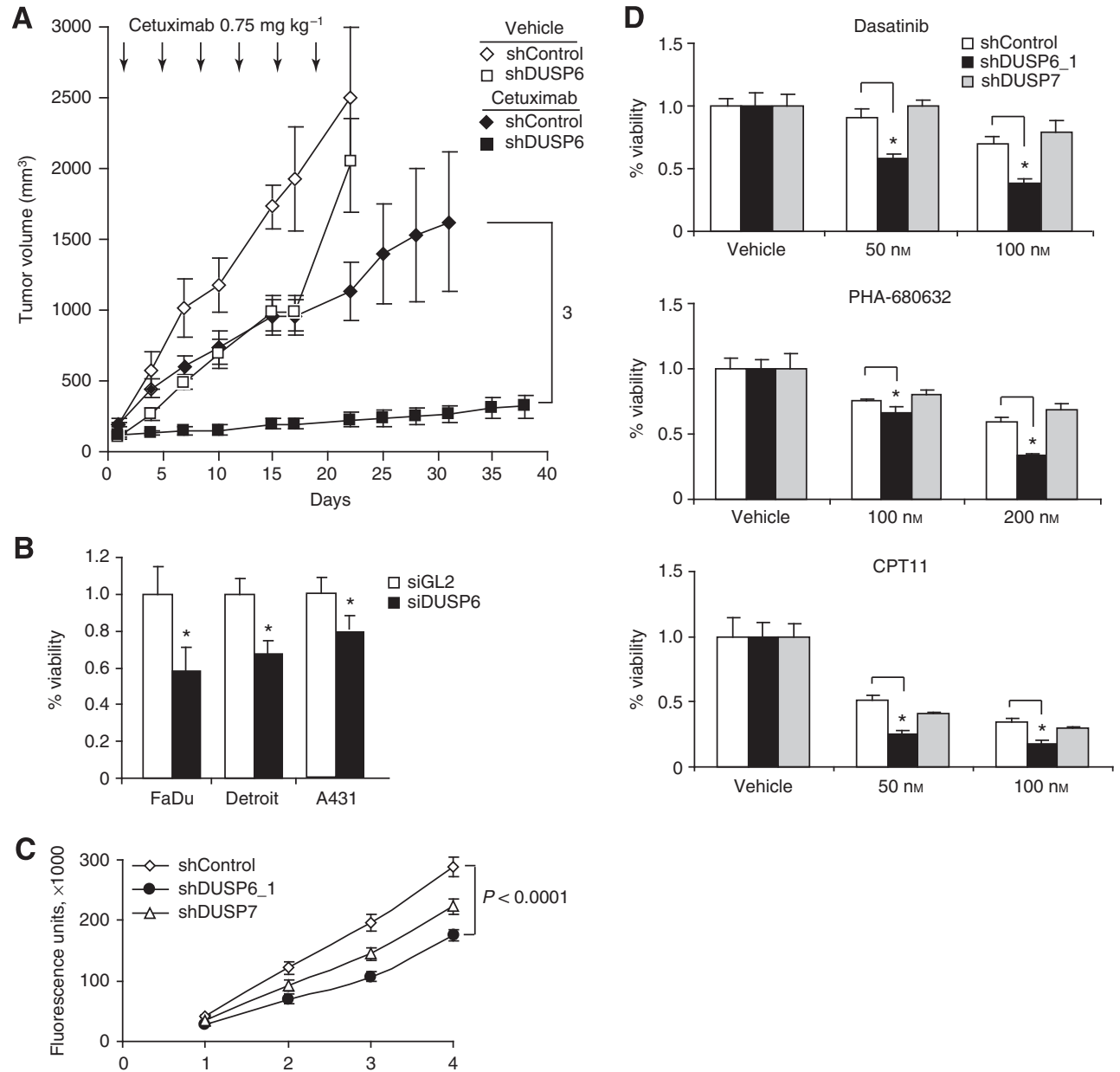

Figure 2. Dual specificity phosphatase 6 regulates growth and sensitivity to multiple cytotoxic agents. (A) Silencing of DUSP6 sensitises A431 xenografts to cetuximab. The results are tumour volumes of xenografts of shRNA-modified A431 cells treated with cetuximab (closed symbols) or vehicle (open symbols); means \pm s.d., $P$-value (3) $<0.0001$. (B) Dual specificity phosphatase 6 depletion decreases the viability of cancer cell lines under basal conditions; the viability (CellTiter Blue) was measured $72 \mathrm{~h}$ after transfection. Shown are mean results of three experiments \pm s.e.m., ${ }^{*} P<0.01$. (C) shDUSP6-silenced A431 cells show slow growth in 10\% FBS-DMEM. Cell numbers were measured using CellTiter Blue assay. (D) Silencing of DUSP6 sensitises A431 cells to inhibitors of the SRC kinase (dasatinib), Aurora kinase (PHA-680632), and a chemotherapy drug (CPT11). Cells were incubated with indicated concentrations for $72 \mathrm{~h}$. Viability data of four independent experiments were normalised to mock-treated cells expressing corresponding shRNA; means \pm s.e.m., shDUSP6 vs non-silencing shRNA control at the corresponding drug concentrations; ${ }^{\star} P<0.05$, the Wilcoxon's test. 
depletion increasing the phosphorylation of ERK1/2 (Camps et al, 1998). We similarly found that EGF positively and erlotinib negatively regulated DUSP6 mRNA, although effects were much less pronounced on the protein level (Supplementary Figure S2B-D). Notably, combined application of erlotinib and siRNA to DUSP6 reduced DUSP6 protein levels more than either treatment alone (Supplementary Figure S1A).

However, in contrast to some previous studies, although DUSP6 was initially identified in our screen based on its role in sensitisation to EGFR inhibitors, our further investigation indicated that depletion of DUSP6 also modestly or significantly reduced the viability of multiple cell lines even without drug treatment (Figure 2B and C). Detailed analysis of A431 carcinoma cells stably depleted of DUSP6 indicated significantly reduced growth both in vitro (Figure 2C) and in vivo (Figure 2A, Supplementary Figure S2A), implying a role for this phosphatase in support of proliferation. This result was surprising, as the increased phosphorylation of ERK1/2 predicted by other studies in this context would be associated with increased proliferation. Indeed, our direct assessment of downstream effectors of EGFR including ERK and AKT showed relatively little effect of depleting DUSP6 in increasing the activity of these proteins (Figure 3 and Supplementary Figure S3). Hence, our data implied that other DUSPs or cellular phosphatases had redundant function for this purpose, whereas DUSP6 depletion might have other critical biological effects. We hence investigated whether depletion of DUSP6 might more uniquely interact with alternative cell signalling pathways.

Dual specificity phosphatase 6 depletion sensitises A431 cells to multiple cytotoxic agents. Next, we examined whether the observed drug sensitisation effect of DUSPP6 depletion was selective to inhibitors of EGFR. We tested sensitivity of shRNAtransduced A431 cells to dasatinib, a multikinase inhibitor ( $>95 \%$ in vitro kinase inhibition of the SRC family, ABL, ACK1, KIT, EPH family, and PDGFR (Anastassiadis et al, 2011)), PHA-680632, an Aurora kinase inhibitor (Soncini et al, 2006), and the DNAdamaging agent CPT11, a topoisomerase 1 inhibitor. ShDUSP6 depletion sensitised A431 cells to each of these agents, indicating lack of specificity for EGFR (Figure 2D). These results again implied a more general function for DUSP6 in cell growth and survival.

Dual specificity phosphatase 6 depletion activates signalling proteins in the DNA damage response (DDR) pathway. To assess the alteration of signalling pathways in drug-sensitised DUSP6-depleted cells in an unbiased fashion, we measured levels of phosphoproteins by solid-phase antibody array. We compared protein phosphorylation in cells made deficient of DUSP6 or
A Signal relative to untreated shControl, $\log _{10}$

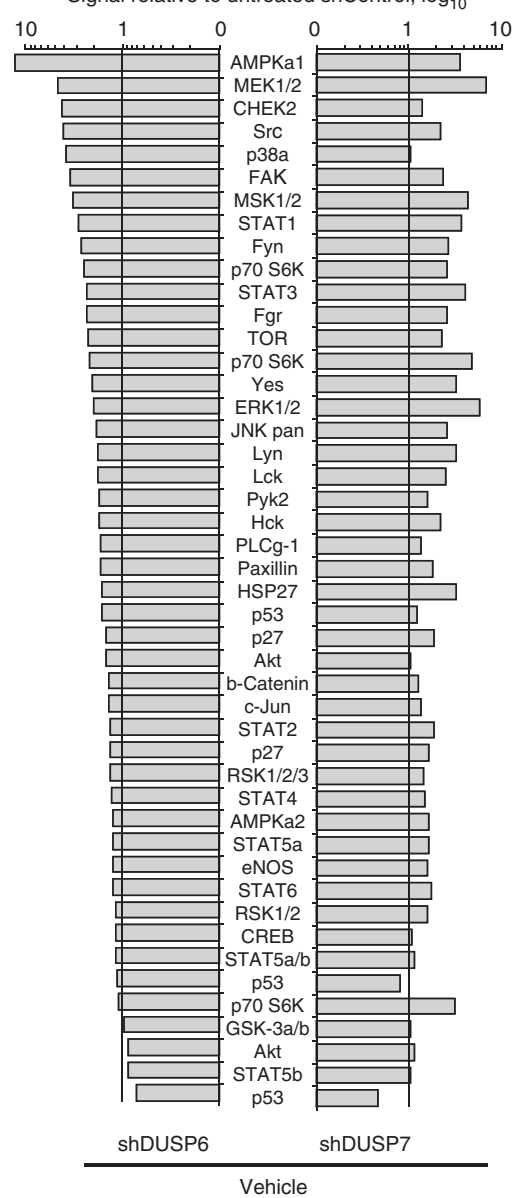

Signal relative to treated shControl, $\log _{10}$

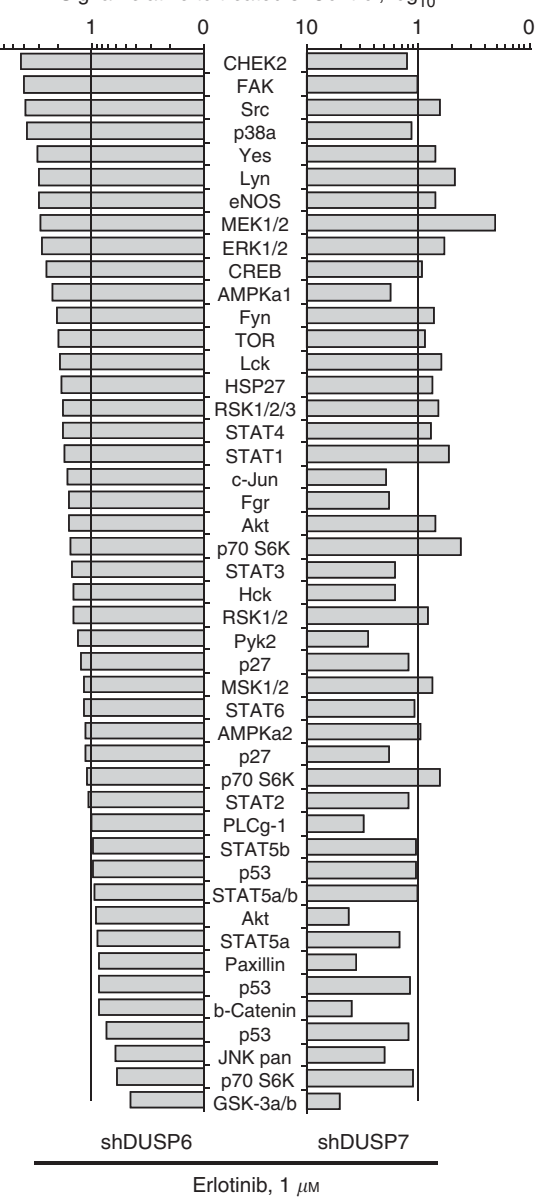

B
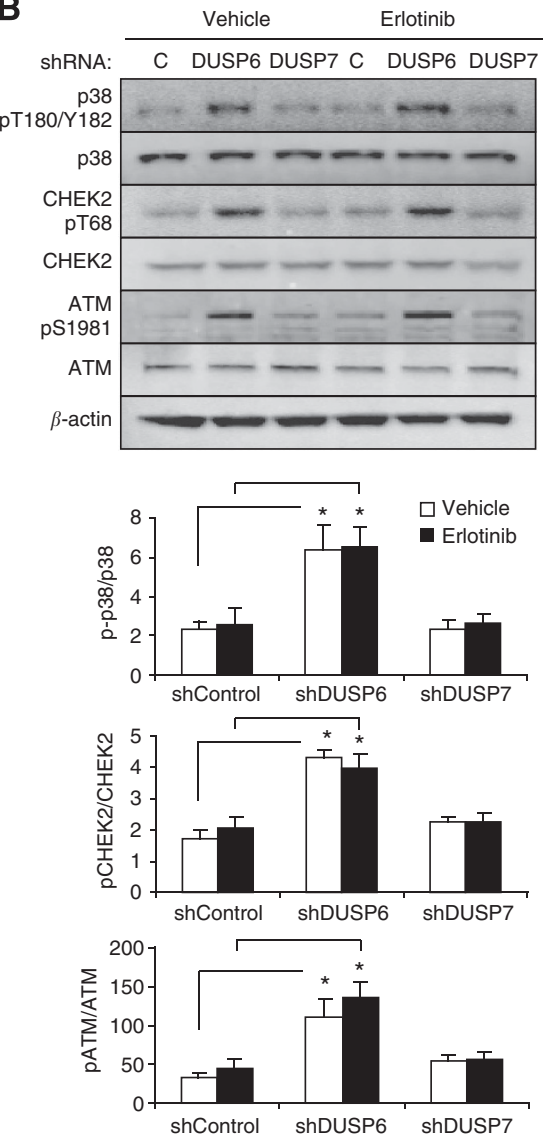

Figure 3. Effects of DUSP6 depletion by phosphoproteomic analysis. (A) Averaged results of reverse-phase antibody array assessment of 46 signalling proteins in A431 cells stably expressing shDUSP6, shDUSP7, or non-silencing shRNA control. Cells were grown in 1\% FBS-DMEM and treated with vehicle (left panel) or $1 \mu \mathrm{M}$ erlotinib (right panel) for $3 \mathrm{~h}$. The data depict relative increase in phosphorylation signal. (B) Western blot analysis of phosphorylation of p38, CHEK2, and their upstream effector ATM. Top, a representative image; bottom, summary of results quantified from the top. Bars represent averaged results of three independent experiments, mean \pm s.e.m. Dual specificity phosphatase 6 -depleted cells vs non-silencing control at the corresponding drug concentrations; ${ }^{\star} P<0.05$, the Wilcoxon's test. 
DUSP7 with specific shRNA, or with a non-targeting control shRNA, in cells grown in $1 \%$ FBS treated for $3 \mathrm{~h}$ with vehicle or $1 \mu \mathrm{M}$ erlotinib (Figure $3 \mathrm{~A}$ ). Following quantification of data, we observed significant increase in phosphorylation relative to control-depleted cells for a limited number of proteins (Figure 3A) in shDUSP6 cells relative to shDUSP7 or control shRNA. Two proteins, the AMP-activated protein kinase AMPKal and the MAPK kinases MEK1/2, were elevated under basal conditions; however, as these changes were similar in both shDUSP6 and shDUSP7 cells, we excluded these proteins from further consideration as relevant to the DUSP6-specific phenotypes affecting cell viability and drug sensitisation. Notably, we again did not observe very significant changes in the phosphorylation status of the EGFR downstream signalling effectors ERK1/2 and AKT (Figure 3A), again suggesting that additional phosphatases compensate for DUSP6 deficiency, and that DUSP6 depletion exerts at least some important biological effects outside of the canonical EGFR pathway.

As candidates for mediators of such effects, we observed that depletion of drug-sensitising DUSP6 but not DUSP7 increased levels of phosphorylated MAPK14/p38 kinase (T180/Y182), and phosphorylated CHEK2 (T68), both in the presence or absence of erlotinib treatment, suggesting they might be relevant to both the sensitisation and the effect on basal cell viability (Figure 3A). These array results were subsequently further confirmed by direct western blotting (Figure 3B), which showed consistently elevated levels of phosphorylation of p38 and CHEK2 in DUSP6-silenced but not in control or DUSP7-depleted cells. Increased phosphop38 (Bulavin et al, 2001; Reinhardt et al, 2007) and phosphoCHEK2 (Reinhardt and Yaffe, 2009) are sensitive biomarkers of cellular DDR (Figure 4A), and are frequently activated in cancer cells. However, no previous study has identified a role for DUSP6 in regulating activation of the DNA repair machinery.

To more broadly explore whether DUSP6 silencing intrinsically activates signalling proteins involved in DDR, and at what level in the pathway DUSP6 might be active, we investigated additional DDR pathway effectors in multiple cancer cell lines (Figures 3 and 4). ATM activation in response to DNA damage (Matsuoka et al, 2007) mediates DNA repair, apoptosis, and cell cycle regulation through phosphorylation of a variety of downstream targets including CHEK2, TP53, 53BP1, and H2AX (Rogakou et al, 1998; Kastan and Lim, 2000; Shiloh, 2006). Silencing of DUSP6 increased the activation-associated S1981 phosphorylation of ATM (Figure 3B) and the S139 phosphorylation of histone H2AX (Supplementary Figure S4) even in the absence of drug treatment, thus suggesting an intrinsic effect of DUSP6 on the DNA repair machinery.

Activation of the DDR pathway in the nuclei is manifested by formation of foci-containing activated DDR proteins (Polo and Jackson, 2011). To address whether DNA repair process is influenced by DUSP6, we assessed DNA breaks by TUNEL assay in cancer cells following siRNA knockdown of DUSP6 and found constitutively higher number of TUNEL-positive cells following DUSP6 depletion (Figure 4B), both in vehicle or CPT11-treated cells, suggesting this response to loss of DUSP6 activity is independent of any exogenous DNA-damaging stimulus. In shDUSP6-depleted A431 cells (Figure 4C and D), we determined an increased formation of nuclear foci containing phosphorylated H2AX, ATM, and 53BP1 compared with controls. These foci were further enhanced by treatment of DUSP6-depleted A431 cells with CPT11 and erlotinib (Figure 4C and D). In accord with decreased viability of multiple carcinoma cell lines following DUSP6 siRNA transfection (Figure $2 \mathrm{~B}$ and $\mathrm{C}$ ), we found consistent upregulation of pH2AX and pATM foci in multiple DUSP6-depleted cancer cell lines, including A431, FaDu, Detroit, and SCC25 (Figure 4E and F), under basal conditions, or following treatment with CPT11 or erlotinib. Furthermore, we used western blot as an alternative method to assess DDR pathway activity (Supplementary Figure S4). Here, silencing of DUSP6 with siRNA in FaDu cells caused a significant increase in $\mathrm{pH} 2 \mathrm{AX}$ under basal conditions as well as following treatment with erlotinib or CPT11. DUSP6 silencing also accentuated CHEK2 phosphorylation, although these changes were less pronounced.

Dual specificity phosphatase 6 depletion induces cell cycle checkpoint delays and increases the intrinsic rate of cellular DNA damage. Persistent presence of DNA breaks typically triggers G1/S checkpoint activation mediated by the ATM/CHEK2 pathway (Zhao et al, 2002) and delays DNA replication in the S phase (Falck et al, 2001). If so, the observed slow growth of xenografts and cell lines in vitro in which DUSP6 was stably depleted with shRNA (Figure 2A and B) might reflect a slowed cell cycle, due to extensive triggering of DNA damage checkpoints. To evaluate the cell cyclespecific effects of DUSP6 loss, we synchronised shRNA-modified A431 cells in the $S$ phase (with thymidine), and then tracked recovery and progression. Following synchronisation in the $S$ phase with thymidine, DUSP6-depleted cells showed a marked delay in the $\mathrm{S}$ phase up to $9 \mathrm{~h}$ after transfer to thymidine-free full growth medium (Figure 5A and B). We observed similar, although less pronounced, delayed progression from the G1 to the S phase in DUSP6-depleted cells (Supplementary Figure S5) using alternative block-release synchronisation techniques (in the $\mathrm{M}$ phase with nocodazole, Supplementary Figure S5A, or in the S phase with CPT11, Supplementary Figure S5B).

Dual specificity phosphatase 6 activity in DDR in the context of MEK to ERK signalling. Given reports of important ERK1/2 functional interactions with DUSP6 (Camps et al, 1998), we considered the possibility that enhanced ERK1/2 activation might influence DUSP6 signalling in the context of DDR. To test this idea, we created a catalytically inactive DUSP6 mutant by replacing the critical cysteine-293 with serine (C293S mutant; Levinthal and Defranco, 2005). For this, we co-transfected HEK-293T cells with plasmids expressing wild-type (WT) or catalytically inactive DUSP6 constructs and a constitutively active form of the ERK1/2 activator MEK-DD (Boehm et al, 2007; Figure 5C and D). Expression of the C293S mutant of DUSP6 independently elevated the basal level of pATM foci-positive cells, similar to the use of a DUSP6 shRNA, implying a dominant-negative activity. By contrast, exogenous expression of WT DUSP6 reduced basal levels of pATM foci by $\sim 50 \%$. In this system, MEK-DD independently caused an increase in the number of ATM-positive foci, accompanied by an induction of ERK phosphorylation (Figure 5C and D). In combination with MEK-DD, the WT DUSP6 eliminated the MEK-DD increase of ATM foci (Figure 5C) and suppressed ERK phosphorylation by $>10$-fold (Figure 5D). In contrast, the C293S mutant of DUSP6 combined with MEK-DD to further increase the number of cells with ATM foci (Figure 5C).

\section{DISCUSSION}

In this study, we found that constitutive or transient ablation of DUSP6 decreased viability and sensitised cells to multiple targeted and untargeted drugs (Figures 1 and 2), increased activity of ATMCHEK2 DDR signalling (Figures 3 and 4), delayed cell cycle progression through S phase (Figure 5, Supplementary Figure S5), and collaborated with MEK1 activation to promote DDR activation. Our data suggest that fine tuning of ERK signalling via DUSP6 may contribute to the regulation of cellular responses to DNA damage induced by chemotherapy in a mechanism involving DUSP6-dependent negative regulation of ATM and CHEK2.

Dual specificity phosphatase 6 has been previously identified as a pro-survival phosphatase (MacKeigan et al, 2005). The expression of DUSP6 is transcriptionally regulated by EGFR activity, and 
A

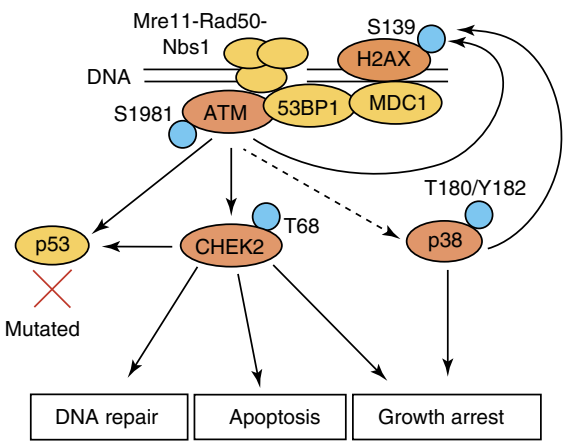

C

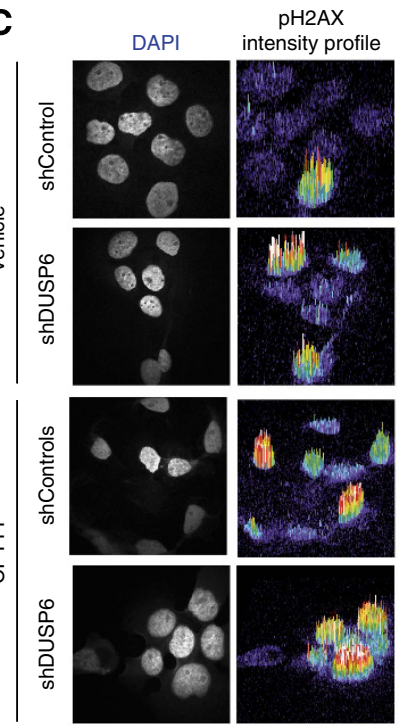

DATM


B

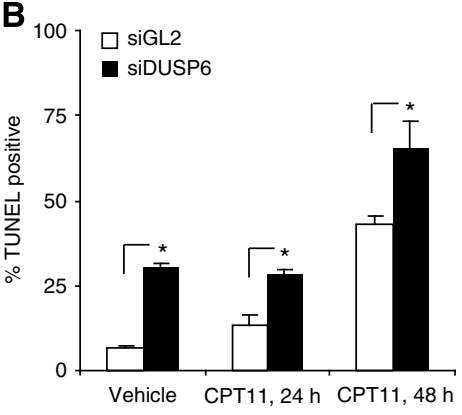


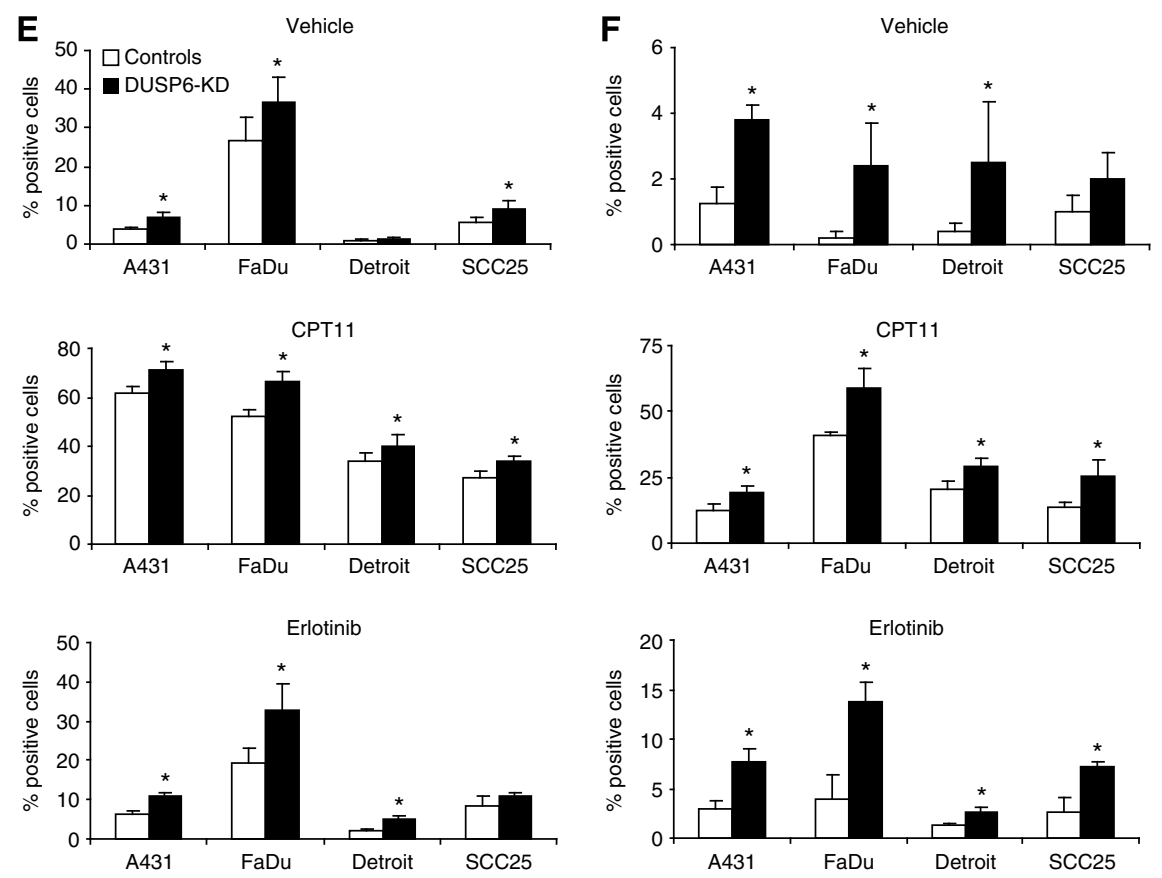

Figure 4. Dual specificity phosphatase 6 deficiency causes intrinsic and drug-stimulated DNA damage. (A) Simplified scheme of the ATM/ CHEK2/p38 pathway. (B) siRNA depletion of DUSP6 in FaDu cells increases DNA breaks by TUNEL Guava flow cytometry assay. siDUSP6 vs siGL2 at the corresponding drug concentrations; ${ }^{*}<0.05$, the Fisher's test. (C) DDR in shRNA-modified A431 cells after overnight treatment with $25 \mathrm{~nm}$ CPT11. (D) Formation of P53BP1 foci in shRNA-modified A431 cells following overnight incubation with vehicle, 25 nм CPT11, or $5 \mu$ m erlotinib. Bars show the mean of three independent experiments \pm s.e.m.; ${ }^{*} P<0.05$, the Fisher's test. (E, F) RNAi depletion of DUSP6 increases pH2AX (E) and PATM (F) foci under basal conditions (1\% FBS-DMEM), and after drug treatment in a panel of cancer cell lines. Silencing of DUSP6 was achieved in A431 with shRNA, other cells lines were transfected with siRNA for $48 \mathrm{~h}$ and treated with $100 \mathrm{~nm}$ CPT11 for $3 \mathrm{~h}$, or $5 \mu \mathrm{M}$ erlotinib for $24 \mathrm{~h}$. Bars represent mean values of three to five independent experiments \pm s.e.m.; $* P<0.05$, the Fisher's test. 
A
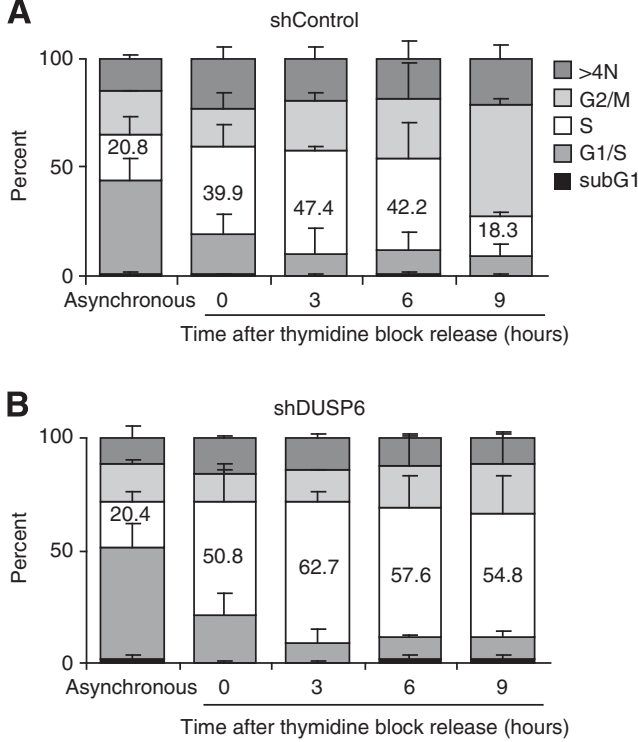
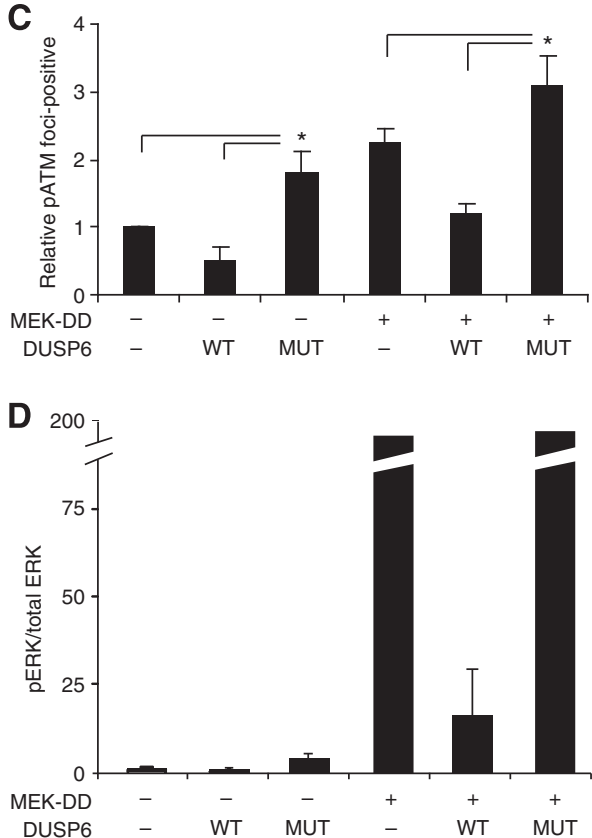

Figure 5. Dual specificity phosphatase 6 depletion delays progression through the $\mathrm{S}$ phase of cell cycle and activates DDR. A431 cells modified with control non-targeting shRNA (A) or targeting DUSP6 (B) were synchronised with $2 \mathrm{~mm}$ thymidine, released from the block, collected after indicated periods of time, and analysed by flow cytometry for DNA contents. In $\mathbf{A}$ and $\mathbf{B}$, graphs represent distribution of cells by different phases of cell cycle at $0,3,6$, and $9 \mathrm{~h}$ after release from thymidine block; the numbers show the percentage of cells in S phase, shControl vs shDUSP6; $P<0.001$ at all time points, the Fisher's test. (C) pATM foci formation assessed by confocal immunofluorescence in HEK-293T cells transfected with pLEX plasmids expressing either wild-type (WT) or C293S mutant (MUT) DUSP6. To activate ERK phosphorylaton, HEK-293T cells were co-transfected with either an empty vector (pBabe) or constitutively active MEK expression plasmid (pBABE-MEK-DD mutant). (D) Western blot assessment of ERK1/2 phosphorylation in HEK-293T cells transfected with DUSP6 and MEK-DD plasmid constructs. In C, D, HEK-293T cells were collected $48 \mathrm{~h}$ after transfection and analysed by immunofluorescence (C) or western blot (D). Shown are the averaged results of three independent experiments; bars, s.d.; ${ }^{\star} P<0.05$, the Student's t-test.

DUSP6 is overexpressed in many cancers harbouring activating mutations of the RAS or BRAF genes (Yun et al, 2009; Degl'Innocenti et al, 2013). Suggestively, DUSP6 overexpression has been associated with cisplatin resistance in non-small cell lung carcinoma (Chen et al, 2007) and tumour growth promotion in glioblastoma (Messina et al, 2011). However, the effects of DUSP6 may be very individually variable depending on the context of associated genomic alterations in a particular cancer type. Given the data presented here, loss of DUSP6 may provide a novel mechanism by which losses of DUSP6 during the pancreatic cancer progression may contribute to accumulation of DNA breaks and lead to genomic instability.

As a general principle, DDR triggers a delay in cell cycle progression to permit DNA repair, or if the damage is too extensive, increased cell death (Reinhardt and Yaffe, 2009). Our results suggest there is a signalling interplay between ATMCHEK2, ERK, and the DUSP6 phosphatase. One possibility might be that an unopposed activity of ERK in the context of DUSP6 deficiency may directly activate the ATM-CHEK2 signalling. Indeed, ERK activity has been shown to regulate cell fate decision following DNA-damaging insults such as ionising radiation or chemotherapy, and forced activation of ERK via MEK1 (Q56P mutant) overexpression sensitised cells to DNA damage-induced apoptosis (Tang et al, 2002; Emery et al, 2009). A recent demonstration of direct interaction between CHEK2 and ERK1/2 in diffuse large B-cell lymphomas (Dai et al, 2011) provides evidence, however, that DUSP6 may be involved in dynamic complexes involving new partners in specific cellular contexts. For instance, direct interaction between ATM, pTP53, and pERK (Heo et al, 2012) exists in a new ternary complex following DNA damage. Along these lines, recent work also demonstrated existence of a ternary complex between DUSP6, ERK2, and p38alpha in which the phosphatase activity of DUSP6 towards p38alpha is allosterically regulated by ERK2 (Zhang et al, 2011). We believe that a simple linear model of RTK-MAPK-DUSP6 regulation may be deficient in not reflecting the abundance of collateral inputs to this conserved pathway (Friedman and Perrimon, 2006). These findings cumulatively suggest that interactions of DUSP6 and its substrates are dynamic and could engage new partners in specific biological processes, or during stress response. Our report provides evidence for the idea that DUSP6 may be a novel and important regulator of cellular responses to DNA damage, with a novel mechanistic explanation for the diverse biological activity of DUSP6 in regulating cancer cell susceptibility to apoptosis and chemotherapy resistance.

\section{ACKNOWLEDGEMENTS}

We are grateful to Dr Emmanuelle Nicolas (Fox Chase Genomics Facility) for quantitative RT-PCR analyses and to Dr Jonathan Chernoff for critical comments on the manuscript. This work was supported by NIH core Grant CA-06927, by the Pew Charitable Fund, and by a generous gift from Mrs Concetta Greenberg to Fox Chase Cancer Center. EAG was supported by NIH R01 CA63366; EAG and IA by Tobacco Settlement funding from the State of Pennsylvania; and IA by NIH K22 CA160725, R21 CA164205 and a career development award from Genentech.

\section{CONFLICT OF INTEREST}

The authors declare no conflict of interest. 


\section{REFERENCES}

Anastassiadis T, Deacon SW, Devarajan K, Ma H, Peterson JR (2011) Comprehensive assay of kinase catalytic activity reveals features of kinase inhibitor selectivity. Nat Biotechnol 29(11): 1039-1045.

Astsaturov I, Ratushny V, Sukhanova A, Einarson MB, Bagnyukova T, Zhou Y, Devarajan K, Silverman JS, Tikhmyanova N, Skobeleva N, Pecherskaya A, Nasto RE, Sharma C, Jablonski SA, Serebriiskii IG, Weiner LM, Golemis EA (2010) Synthetic lethal screen of an EGFRcentered network to improve targeted therapies. Sci Signal 3(140): ra67.

Bluthgen N, Legewie S, Kielbasa SM, Schramme A, Tchernitsa O, Keil J, Solf A, Vingron M, Schafer R, Herzel H, Sers C (2009) A systems biological approach suggests that transcriptional feedback regulation by dualspecificity phosphatase 6 shapes extracellular signal-related kinase activity in RAS-transformed fibroblasts. FEBS J 276(4): 1024-1035.

Boehm JS, Zhao JJ, Yao J, Kim SY, Firestein R, Dunn IF, Sjostrom SK, Garraway LA, Weremowicz S, Richardson AL, Greulich H, Stewart CJ, Mulvey LA, Shen RR, Ambrogio L, Hirozane-Kishikawa T, Hill DE, Vidal M, Meyerson M, Grenier JK, Hinkle G, Root DE, Roberts TM, Lander ES, Polyak K, Hahn WC (2007) Integrative genomic approaches identify IKBKE as a breast cancer oncogene. Cell 129(6): 1065-1079.

Bulavin DV, Higashimoto Y, Popoff IJ, Gaarde WA, Basrur V, Potapova O, Appella E, Fornace Jr AJ (2001) Initiation of a G2/M checkpoint after ultraviolet radiation requires p38 kinase. Nature 411(6833): 102-107.

Camps M, Nichols A, Gillieron C, Antonsson B, Muda M, Chabert C, Boschert U, Arkinstall S (1998) Catalytic activation of the phosphatase MKP-3 by ERK2 mitogen-activated protein kinase. Science 280(5367): $1262-1265$.

Chen HY, Yu SL, Chen CH, Chang GC, Chen CY, Yuan A, Cheng CL, Wang CH, Terng HJ, Kao SF, Chan WK, Li HN, Liu CC, Singh S, Chen WJ, Chen JJ, Yang PC (2007) A five-gene signature and clinical outcome in non-small-cell lung cancer. $N$ Engl J Med 356(1): 11-20.

Dai B, Zhao XF, Mazan-Mamczarz K, Hagner P, Corl S, Bahassi el M, Lu S, Stambrook PJ, Shapiro P, Gartenhaus RB (2011) Functional and molecular interactions between ERK and CHK2 in diffuse large B-cell lymphoma. Nat Commun 2: 402.

De Roock W, Janssens M, Biesmans B, Jacobs B, De Schutter J, Fieuws S, Van Cutsem E, Tejpar S (2009) DUSPs as markers of MEK/Erk activation in primary colorectal cancer. J Clin Oncol 27(15S): 4064.

Degl'Innocenti D, Romeo P, Tarantino E, Sensi M, Cassinelli G, Catalano V, Lanzi C, Perrone F, Pilotti S, Seregni E, Pierotti MA, Greco A, Borrello MG (2013) DUSP6/MKP3 is overexpressed in papillary and poorly differentiated thyroid carcinoma and contributes to neoplastic properties of thyroid cancer cells. Endocrine-related Cancer 20(1): 23-37.

Dowd S, Sneddon AA, Keyse SM (1998) Isolation of the human genes encoding the pyst1 and Pyst2 phosphatases: characterisation of Pyst2 as a cytosolic dual-specificity MAP kinase phosphatase and its catalytic activation by both MAP and SAP kinases. J Cell Sci 111, Pt 22 3389-3399.

Eblaghie MC, Lunn JS, Dickinson RJ, Munsterberg AE, Sanz-Ezquerro JJ, Farrell ER, Mathers J, Keyse SM, Storey K, Tickle C (2003) Negative feedback regulation of FGF signaling levels by Pyst1/MKP3 in chick embryos. Curr Biol 13(12): 1009-1018.

Emery CM, Vijayendran KG, Zipser MC, Sawyer AM, Niu L, Kim JJ, Hatton C, Chopra R, Oberholzer PA, Karpova MB, MacConaill LE, Zhang J, Gray NS, Sellers WR, Dummer R, Garraway LA (2009) MEK1 mutations confer resistance to MEK and B-RAF inhibition. Proc Natl Acad Sci USA 106(48): 20411-20416.

Falck J, Mailand N, Syljuasen RG, Bartek J, Lukas J (2001) The ATM-Chk2Cdc25A checkpoint pathway guards against radioresistant DNA synthesis. Nature 410(6830): 842-847.

Friedman A, Perrimon N (2006) A functional RNAi screen for regulators of receptor tyrosine kinase and ERK signalling. Nature 444(7116): 230-234.

Furukawa T (2009) Molecular pathology of pancreatic cancer: implications for molecular targeting therapy. Clin Gastroenterol Hepatol 7(11 Suppl): S35-S39.

Furukawa T, Sunamura M, Motoi F, Matsuno S, Horii A (2003) Potential tumor suppressive pathway involving DUSP6/MKP-3 in pancreatic cancer. Am J Pathol 162(6): 1807-1815.
Heo JI, Oh SJ, Kho YJ, Kim JH, Kang HJ, Park SH, Kim HS, Shin JY, Kim MJ, Kim M, Kim SC, Park JB, Kim J, Lee JY (2012) ATM mediates interdependent activation of $\mathrm{p} 53$ and ERK through formation of a ternary complex with p-p53 and p-ERK in response to DNA damage. Mol Biol Rep 39(8): 8007-8014.

Kastan MB, Lim DS (2000) The many substrates and functions of ATM. Nat Rev Mol Cell Biol 1(3): 179-186.

Keyse SM (2008) Dual-specificity MAP kinase phosphatases (MKPs) and cancer. Cancer Metastasis Rev 27(2): 253-261.

Levinthal DJ, Defranco DB (2005) Reversible oxidation of ERK-directed protein phosphatases drives oxidative toxicity in neurons. J Biol Chem 280(7): 5875-5883.

Li C, Scott DA, Hatch E, Tian X, Mansour SL (2007) Dusp6 (Mkp3) is a negative feedback regulator of FGF-stimulated ERK signaling during mouse development. Development 134(1): 167-176.

MacKeigan JP, Murphy LO, Blenis J (2005) Sensitized RNAi screen of human kinases and phosphatases identifies new regulators of apoptosis and chemoresistance. Nat Cell Biol 7(6): 591-600.

Matsuoka S, Ballif BA, Smogorzewska A, McDonald 3rd ER, Hurov KE, Luo J, Bakalarski CE, Zhao Z, Solimini N, Lerenthal Y, Shiloh Y, Gygi SP, Elledge SJ (2007) ATM and ATR substrate analysis reveals extensive protein networks responsive to DNA damage. Science 316(5828): 1160-1166.

Messina S, Frati L, Leonetti C, Zuchegna C, Di Zazzo E, Calogero A, Porcellini A (2011) Dual-specificity phosphatase DUSP6 has tumor-promoting properties in human glioblastomas. Oncogene 30(35): 3813-3820.

Polo SE, Jackson SP (2011) Dynamics of DNA damage response proteins at DNA breaks: a focus on protein modifications. Genes Dev 25(5): 409-433.

Reinhardt HC, Aslanian AS, Lees JA, Yaffe MB (2007) p53-deficient cells rely on ATM- and ATR-mediated checkpoint signaling through the p38MAPK/MK2 pathway for survival after DNA damage. Cancer Cell 11(2): 175-189.

Reinhardt HC, Yaffe MB (2009) Kinases that control the cell cycle in response to DNA damage: Chk1, Chk2, and MK2. Curr Opin Cell Biol 21(2): 245-255.

Rogakou EP, Pilch DR, Orr AH, Ivanova VS, Bonner WM (1998) DNA double-stranded breaks induce histone H2AX phosphorylation on serine 139. J Biol Chem 273(10): 5858-5868.

Shiloh Y (2006) The ATM-mediated DNA-damage response: taking shape. Trends Biochem Sci 31(7): 402-410.

Soncini C, Carpinelli P, Gianellini L, Fancelli D, Vianello P, Rusconi L, Storici P, Zugnoni P, Pesenti E, Croci V, Ceruti R, Giorgini ML, Cappella P, Ballinari D, Sola F, Varasi M, Bravo R, Moll J (2006) PHA-680632, a novel Aurora kinase inhibitor with potent antitumoral activity. Clin Cancer Res 12(13): 4080-4089.

Tang D, Wu D, Hirao A, Lahti JM, Liu L, Mazza B, Kidd VJ, Mak TW, Ingram AJ (2002) ERK activation mediates cell cycle arrest and apoptosis after DNA damage independently of p53. J Biol Chem 277(15): 12710-12717.

Yun J, Rago C, Cheong I, Pagliarini R, Angenendt P, Rajagopalan H, Schmidt K, Willson JK, Markowitz S, Zhou S, Diaz Jr. LA, Velculescu VE, Lengauer C, Kinzler KW, Vogelstein B, Papadopoulos N (2009) Glucose deprivation contributes to the development of KRAS pathway mutations in tumor cells. Science 325(5947): 1555-1559.

Zhang YY, Wu JW, Wang ZX (2011) Mitogen-activated protein kinase (MAPK) phosphatase 3-mediated cross-talk between MAPKs ERK2 and p38alpha. J Biol Chem 286(18): 16150-16162.

Zhang Z, Kobayashi S, Borczuk AC, Leidner RS, Laframboise T, Levine AD, Halmos B (2010) Dual specificity phosphatase 6 (DUSP6) is an ETS-regulated negative feedback mediator of oncogenic ERK signaling in lung cancer cells. Carcinogenesis 31(4): 577-586.

Zhao H, Watkins JL, Piwnica-Worms H (2002) Disruption of the checkpoint kinase 1/cell division cycle 25A pathway abrogates ionizing radiation-induced S and G2 checkpoints. Proc Natl Acad Sci USA 99(23): 14795-14800

This work is published under the standard license to publish agreement. After 12 months the work will become freely available and the license terms will switch to a Creative Commons AttributionNonCommercial-Share Alike 3.0 Unported License.

Supplementary Information accompanies this paper on British Journal of Cancer website (http://www.nature.com/bjc) 